\title{
Geometric Interpolation by Quartic Rational Spline Motions
}

\author{
B. Jüttler ${ }^{1}$, M. Krajnc ${ }^{2}$ and E. Žagar ${ }^{2}$ \\ ${ }^{1}$ Institute of Applied Geometry, Johannes Kepler University, Linz, Austria, e-mail: \\ bert.juettler@jku.at \\ ${ }^{2}$ IMFM and FMF, University of Ljubljana, Slovenia. e-mail: \\ marjeta.krajnc@fmf.uni-lj.si,emil.zagar@fmf.uni-lj.si
}

\begin{abstract}
We discuss piecewise rational motions with first order geometric continuity. In addition we describe an interpolation scheme generating rational spline motions of degree four matching given positions which are partially complemented by associated tangent information. As the main advantage of using geometric interpolation, it makes it possible to deal successfully with the unequal distribution of degrees of freedom between the trajectory of the origin and the rotation part of the motion.
\end{abstract}

Key words: motion design, geometric interpolation, rational spline motion, geometric continuity

\section{Introduction}

Geometric interpolation techniques [1] generate spline curves from given geometric data, such as points, tangents, and perhaps even curvature information. As an advantage, they generally require lower polynomial degrees than standard methods. For instance, a polynomial cubic in the plane can match two points with associated tangents and curvatures [2], while the interpolation of two points with associated first and second derivatives needs curves of degree five.

Geometric interpolation techniques are closely related to the concept of geometric continuity, which generalizes the notion of derivative continuity by eliminating the influence of the parameterization [3]. The present paper introduces the new notion of geometrically continuous rational spline motions and describes a first geometric interpolation method for motion design using piecewise quartic motions.

Bennett biarcs, which form another subset of the class of quartic rational spline motions, have recently been used for geometrically interpolating two positions with associated tangent information [4]. It was observed that the collisions between fixed planes and moving points (and similar for fixed points and moving planes) can be detected simply by solving quartic polynomials. However, as a disadvantage, the class of motions described by Bennett biarcs cannot handle motions with changing chirality (i.e., orientation of the instantaneous screw motions). 
The method described in this paper uses two segments of rational quartic motions for interpolating three positions, two of which are complemented by associated tangent information. Compared to Bennett biarcs, they can handle one additional position, and no problems with the chirality of the motion are present. Still, the collision between moving points and fixed planes leads simply to quartic polynomials, while this is no longer true for collisions between moving planes and fixed points.

The remainder of this paper is organized as follows. In Section 2 geometrically continuous rational spline motions are presented. The next section introduces the interpolation problem for quartic rational spline motion, and Section 4 provides its explicit construction together with some numerical examples. The paper is concluded by some ideas for the future work.

\section{Geometrically continuous rational spline motions}

A rigid body motion is described by the trajectory $\mathbf{v}(t)=\left(v_{1}(t), v_{2}(t), v_{3}(t)\right)^{\top}$ of the origin of the moving system and by the $3 \times 3$ rotation matrix $R(t)$. Using quaternions $\mathbf{q}=\left(q_{0}, q_{1}, q_{2}, q_{3}\right)^{\top}$, the rotation matrix $R$ can be represented by

$$
R=\frac{1}{q_{0}^{2}+q_{1}^{2}+q_{2}^{2}+q_{3}^{2}}\left[\begin{array}{ccc}
q_{0}^{2}+q_{1}^{2}-q_{2}^{2}-q_{3}^{2} & 2\left(q_{1} q_{2}-q_{0} q_{3}\right) & 2\left(q_{1} q_{3}+q_{0} q_{2}\right) \\
2\left(q_{1} q_{2}+q_{0} q_{3}\right) & q_{0}^{2}-q_{1}^{2}+q_{2}^{2}-q_{3}^{2} & 2\left(q_{2} q_{3}-q_{0} q_{1}\right) \\
2\left(q_{1} q_{3}-q_{0} q_{2}\right) & 2\left(q_{2} q_{3}+q_{0} q_{1}\right) & q_{0}^{2}-q_{1}^{2}-q_{2}^{2}+q_{3}^{2}
\end{array}\right]
$$

see [5]. The trajectory of an arbitrary point $\widehat{\mathbf{p}}$ of the moving system is

$$
\mathbf{p}(t)=\mathbf{v}(t)+R(t) \widehat{\mathbf{p}}
$$

In particular we are interested in rational spline motions which are obtained by choosing rational spline (i.e., piecewise rational) functions $q_{i}(t)$ and $v_{i}(t)$ representing the coordinates of the quaternion and of the trajectory.

Rational motions can be classified by the degree of their trajectories, which is called the degree of the motion, see [6, 7]. In particular, by considering quadratic polynomials $q_{i}(t)$ one obtains rational motions of degree four or higher. In order to obtain rational motions of degree four, the three functions $v_{i}$ should be chosen as

$$
v_{i}=\frac{w_{i}}{q_{0}^{2}+q_{1}^{2}+q_{2}^{2}+q_{3}^{2}}, \quad i=1,2,3,
$$

where $w_{i}$ are quartic polynomials.

Note that there is a remarkable discrepancy in the number of degrees of freedom (i.e., free coefficients) which can be used for specifying the rotation matrix and the trajectory of the origin. The rotation matrix of a quartic motion of this type is controlled by 11 free parameters ( 4 (number of $q_{i}$ ) $\times 3$ (number of coefficients per quadratic polynomial $q_{i}$ ) -1 (normalization)). On the other hand, the trajectory of 
the origin possesses $3 \times 5=15$ degrees of freedom. Consequently, the trajectory of the origin is far more flexible than the rotation part.

This discrepancy becomes even larger for motions of higher degree. In order to obtain a motion of degree $2 d$, the quaternion can be chosen as a polynomial of degree $d$ with $4(d+1)$ coefficients (where one of them can be eliminated by a normalization). The trajectory of the origin, constructed according to (2), then provides $3(2 d+1)=6 d+3$ degrees of freedom.

Consider a rational spline curve $\mathbf{p}(t)$ with domain $I=[0, M]$, where $M$ is the number of segments of the curve, and knots (i.e., segment end points) at the integers. This curve is said to be geometrically smooth of first order (also called $G^{1}$ or tangent continuous) if it is continuous $\left(C^{0}\right)$ and satisfies

$$
\forall s \in I^{\circ} \quad \exists \lambda(s)>0: \quad \lim _{t \downarrow s} \mathbf{p}^{\prime}(t)=\lambda(s) \lim _{t \uparrow s} \mathbf{p}^{\prime}(t),
$$

where $I^{\circ}$ denotes the interior of the parameter interval $I$. In other words, the curve has a well-defined unit tangent vector everywhere. The proportionality factor $\lambda$ is equal to 1 everywhere, except for the knots, where it is assumed to be positive.

We say that a rational spline motion is $G^{1}$ smooth if all its trajectories (1) are $G^{1}$ continuous, where additionally the proportionality factors $\lambda$ are independent of $\hat{\mathbf{p}}$. This is the case if and only if the curve in 12-dimensional space which is defined by the components of $\mathbf{v}(t)$ and $R(t)$ is $G^{1}$ continuous. This curve can be considered as the image of the motion under the kinematic mapping studied in [8]. All point trajectories are obtained as images of this curve under affine mappings.

\section{Interpolation problem}

In the remainder of this paper we consider the following geometric interpolation problem in motion design. For $2 m+1$ given positions, each of which is represented by the coordinates $\mathbf{C}_{j}$ of the origin of the coordinate system along with a normalized quaternion $\mathbf{Q}_{j}$ (considered as fourdimensional vector), $j=0, \ldots 2 m$, find a $G^{1}$ smooth rational spline motion which interpolates these positions. Moreover, we assume that every second position (with an even index $j$ ) is complemented by associated derivative information $\mathbf{t}_{j}$ for the motion of the origin and by $\mathbf{u}_{j}$ for the quaternions. This additional derivative information is either specified by the user or it can be estimated from the data, e.g., using the methods described in [9].

Note that each rotation can be represented by two normalized quaternions, which differ by the sign of their components. We choose the signs so that the standard inner product $\mathbf{Q}_{j}^{\top} \mathbf{Q}_{j+1}$ in $\mathbb{R}^{4}$ is positive.

More precisely, we are looking for seven continuous rational spline functions $\mathbf{v}=\left(v_{1}, v_{2}, v_{3}\right)^{\top}$ and $\mathbf{q}=\left(q_{0}, q_{1}, q_{2}, q_{3}\right)^{\top}$ with domain $I=[0,2 m]$ which satisfy the position interpolation conditions

$$
\mathbf{v}(j)=\mathbf{C}_{j}, \quad \mathbf{q}(j)=\rho_{j} \mathbf{Q}_{j}, \quad j=0, \ldots, 2 m,
$$


the tangent interpolation conditions

$$
\lim _{t \downarrow 2 k} \mathbf{v}^{\prime}(t)=\sigma_{k} \mathbf{t}_{2 k}, \quad \lim _{t \downarrow 2 k} \mathbf{q}^{\prime}(t)=\sigma_{k} \rho_{2 k} \mathbf{u}_{2 k}, \quad k=0, \ldots, m
$$

where for $t=2 m$ the limits from the left must be used, and the $G^{1}$ conditions

$$
\lim _{t \downarrow j} \mathbf{v}^{\prime}(t)=\lambda_{j} \lim _{t \uparrow j} \mathbf{v}^{\prime}(t), \quad \lim _{t \downarrow j} \mathbf{q}^{\prime}(t)=\lambda_{j} \lim _{t \uparrow j} \mathbf{q}^{\prime}(t), \quad j=1, \ldots, 2 m-1 .
$$

The scaling factors $\rho_{j}, \sigma_{k}$ and $\lambda_{j}$ are unknown and should all be positive.

It should be noted that the $G^{1}$ conditions are sufficient, but not necessary for the motion to be smooth. Indeed, they guarantee that the quaternion curve $\mathbf{q}(t)$ is $G^{1}$, but also certain non- $G^{1}$-smooth quaternion curves may give tangent continuous rational motions. Currently we are not aware of any sensible use of these additional degrees of freedom, and we therefore decided not to use them.

\section{Construction of quartic rational spline motions}

In this section, the technique of parabolic geometric interpolation in $\mathbb{R}^{3}$ will be used to construct quartic rational spline motions, see [10] and [11] for the related discussion in the space of curves. Since geometric interpolation schemes depend on the dimension of the space, the quaternion data will be transformed to a particular three-dimensional subspace, without changing the geometric meaning.

Consider first the case with only two segments, i.e., $m=1$. We start with the construction of the spherical part of the motion. Recall that proportional quaternions represent the same rotation. We choose $\rho_{0}=\rho_{2}=1$. By the QR decomposition

$$
\left[\mathbf{u}_{0}, \mathbf{Q}_{2}-\mathbf{Q}_{0}, \mathbf{u}_{2}\right]=F U, \quad F:=\left[\mathbf{f}_{1}, \mathbf{f}_{2}, \mathbf{f}_{3}\right] \in \mathbb{R}^{4 \times 3}, \quad U \in \mathbb{R}^{3 \times 3},
$$

where $F^{\top} F=I$ and $U$ is an upper triangular matrix, we obtain an orthonormal basis $\left\{\mathbf{f}_{i}\right\}_{i=1}^{3}$ of the threedimensional hyperplane $\mathscr{S}$ spanned by $\mathbf{u}_{0}, \mathbf{Q}_{2}-\mathbf{Q}_{0}$ and $\mathbf{u}_{2}$.

In order to work in this threedimensional hyperplane, we must choose $\rho_{1}$ so that $\rho_{1} \mathbf{Q}_{1}-\mathbf{Q}_{0} \in \mathscr{S}$ too. If $\mathbf{Q}_{1}-\mathbf{Q}_{0}$ is already in $\mathscr{S}$, then $\rho_{1}=1$, otherwise

$$
\rho_{1}=\frac{\operatorname{det}\left(\mathbf{f}_{1}, \mathbf{f}_{2}, \mathbf{f}_{3}, \mathbf{Q}_{0}\right)}{\operatorname{det}\left(\mathbf{f}_{1}, \mathbf{f}_{2}, \mathbf{f}_{3}, \mathbf{Q}_{1}\right)} .
$$

Obviously $\rho_{1}$ will be positive if and only if both determinants in (6) have the same sign.

In the next step, we construct a parabolic $G^{1}$ polynomial spline $\mathbf{q}$ with knots $0,1,2$ satisfying

$$
\mathbf{q}(2 j)=\mathbf{Q}_{2 j}, \quad \mathbf{q}^{\prime}(2 j)=\sigma_{j} \mathbf{u}_{2 j}, \quad j=0,1,
$$

and 


$$
\mathbf{q}(1)=\rho_{1} \mathbf{Q}_{1}, \quad \lim _{t \downarrow 1} \mathbf{q}^{\prime}(t)=\lambda_{1} \lim _{t \uparrow 1} \mathbf{q}^{\prime}(t) .
$$

Clearly, the standard parabolic interpolation scheme in $\mathbb{R}^{3}$ does not provide enough freedom to solve this problem, thus the geometric interpolation technique must be applied (for general geometric methods see e.g. [1]). Let $\mathbf{q}_{j}:=\left.\mathbf{q}\right|_{[j, j+1]}, j=0,1$, be the quadratic polynomial pieces of $\mathbf{q}$ (segments of parabolas). They can be written in Bernstein-Bézier form as

$$
\begin{aligned}
& \mathbf{q}_{0}(t):=\mathbf{Q}_{0} B_{2,0}(t)+\mathbf{Q}_{01} B_{2,1}(t)+\rho_{1} \mathbf{Q}_{1} B_{2,2}(t), \\
& \mathbf{q}_{1}(t):=\rho_{1} \mathbf{Q}_{1} B_{2,0}(t-1)+\mathbf{Q}_{12} B_{2,1}(t-1)+\mathbf{Q}_{2} B_{2,2}(t-1),
\end{aligned}
$$

where $B_{n, i}(t)=\left(\begin{array}{c}n \\ i\end{array}\right) t^{i}(1-t)^{n-i}$ are the Bernstein basis polynomials of degree $n$. Basic properties of Bézier curves (see e.g. [3]) imply

$$
\mathbf{q}_{0}^{\prime}(0)=2\left(\mathbf{Q}_{01}-\mathbf{Q}_{0}\right), \quad \mathbf{q}_{1}^{\prime}(2)=2\left(\mathbf{Q}_{2}-\mathbf{Q}_{12}\right) .
$$

Now (9) and (10), along with the interpolation conditions (7), lead to

$$
\mathbf{Q}_{01}=\mathbf{Q}_{0}+\frac{1}{2} \sigma_{0} \mathbf{u}_{0}, \quad \mathbf{Q}_{12}=\mathbf{Q}_{2}-\frac{1}{2} \sigma_{2} \mathbf{u}_{2}
$$

The $G^{1}$ condition (8) can be rewritten as

$$
\mathbf{Q}_{12}-\rho_{1} \mathbf{Q}_{1}=\lambda_{1}\left(\rho_{1} \mathbf{Q}_{1}-\mathbf{Q}_{01}\right)
$$

and (11) finally gives the nonlinear system

$$
-\frac{1}{2} \lambda_{1} \sigma_{0} \mathbf{u}_{0}+\lambda_{1} \Delta \mathbf{Q}_{0}+\frac{1}{2} \sigma_{2} \mathbf{u}_{2}=\Delta \mathbf{Q}_{1}
$$

for $\sigma_{0}, \sigma_{1}, \lambda_{1}$, where $\Delta \mathbf{Q}_{0}:=\rho_{1} \mathbf{Q}_{1}-\mathbf{Q}_{0}$ and $\Delta \mathbf{Q}_{1}:=\mathbf{Q}_{2}-\rho_{1} \mathbf{Q}_{1}$. Fortunately, this system can be transformed into to the linear form

$$
F^{\top}\left[-\frac{1}{2} \mathbf{u}_{0}, \Delta \mathbf{Q}_{0}, \frac{1}{2} \mathbf{u}_{2}\right]\left[\begin{array}{c}
\lambda_{1} \sigma_{0} \\
\lambda_{1} \\
\sigma_{2}
\end{array}\right]=F^{\top} \Delta \mathbf{Q}_{1},
$$

and Cramer's rule yields

$$
\sigma_{0}=2 \frac{D_{1}}{D_{2}}, \quad \sigma_{2}=2 \frac{D_{4}}{D_{3}}, \quad \lambda_{1}=\frac{D_{2}}{D_{3}},
$$

where $D:=F^{\top}\left[\mathbf{u}_{0}, \Delta \mathbf{Q}_{0}, \Delta \mathbf{Q}_{1}, \mathbf{u}_{2}\right]$, and $D_{i}$ is the determinant of the submatrix of $D$ with $i$-th column omitted.

The sufficient and necessary conditions for $\sigma_{0}, \sigma_{2}$ and $\lambda_{1}$ to be positive is that all determinants $D_{i}, i=1,2,3,4$, have the same sign.

There is also a simple geometric interpretation of this solution (see Figure 1). Let $\Sigma_{0}$ be the plane spanned by $\rho_{1} \mathbf{Q}_{1}$ and the line $\ell_{0}$ through $\mathbf{Q}_{0}$ in the direction of $\mathbf{u}_{0}$. 
Further, let $\Sigma_{1}$ be another plane defined by $\rho_{1} \mathbf{Q}_{1}$ and the line $\ell_{1}$ through $\mathbf{Q}_{2}$ in the direction of $\mathbf{u}_{2}$. This two planes determine the line which intersects $\ell_{0}$ and $\ell_{1}$ in $\mathbf{Q}_{01}$ and $\mathbf{Q}_{12}$, respectively. The solution is admissible if this two intersections lie on the proper sides of the points $\mathbf{Q}_{0}$ and $\mathbf{Q}_{2}$.

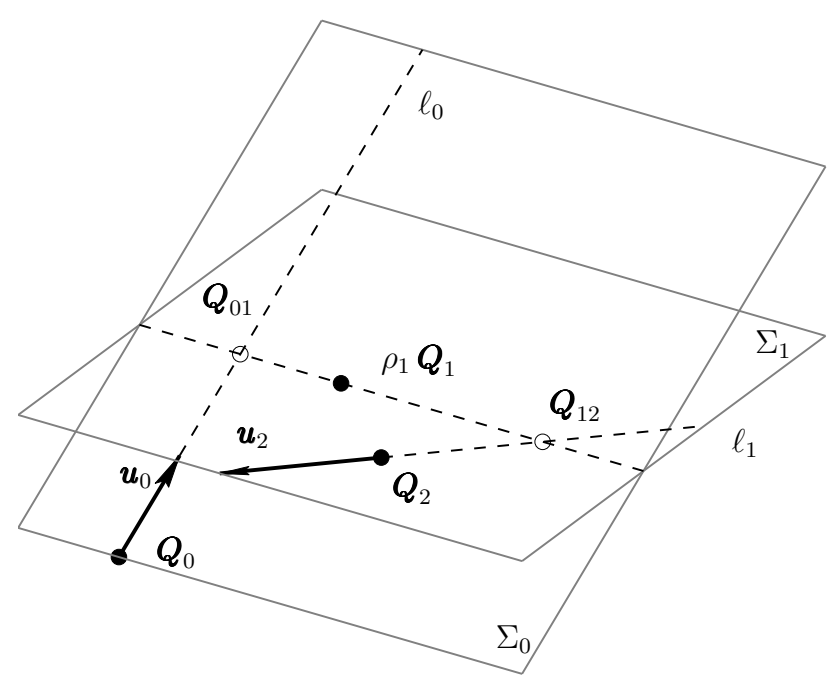

Fig. 1 Geometric construction of control points $\mathbf{Q}_{01}$ and $\mathbf{Q}_{12}$ given by (11).

We are now left to construct the trajectory $\mathbf{v}$ of the origin of the moving coordinate system. Let $\mathbf{v}$ be composed of two quartic rational spline curves $\mathbf{v}_{j}$,

$$
\mathbf{v}_{j}:=\left.\mathbf{v}\right|_{[j, j+1]}, \quad \mathbf{v}_{j}=\frac{\mathbf{w}_{j}}{r_{j}}, \quad r_{j}:=\left\|\mathbf{q}_{j}\right\|^{2}, \quad j=0,1,
$$

where $\mathbf{w}_{0}, \mathbf{w}_{1}$ are the unknown polynomial curves of degree $\leq 4$, and $\mathbf{q}_{0}, \mathbf{q}_{1}$ are obtained from the interpolation of the rotational part. Note that the parameters $\sigma_{0}, \sigma_{1}, \lambda_{1}$ are already fixed by (12). The interpolation conditions (3), (4) and (5) clearly do not provide sufficiently many equations to uniquely determine $\mathbf{w}_{0}, \mathbf{w}_{1}$. One way to use the additional freedom is to to restrict $\mathbf{w}_{j}$ to a cubic polynomial and prescribe also the tangent direction $\mathbf{t}_{1}$ at $\mathbf{C}_{1}$. Then a standard cubic interpolation scheme can be applied to determine $\mathbf{w}_{j}, j=0,1$, that satisfy

$$
\mathbf{w}_{j}(k)=r_{j}(k) \mathbf{C}_{k}, \quad k=j, j+1, \quad j=0,1,
$$

and

$$
\begin{aligned}
& \mathbf{w}_{0}^{\prime}(0)=r_{0}(0) \sigma_{0} \mathbf{t}_{0}+r_{0}^{\prime}(0) \mathbf{C}_{0}, \quad \mathbf{w}_{0}^{\prime}(1)=r_{0}(1) \mathbf{t}_{1}+r_{0}^{\prime}(1) \mathbf{C}_{1}, \\
& \mathbf{w}_{1}^{\prime}(1)=\lambda_{1} r_{1}(1) \mathbf{t}_{1}+r_{1}^{\prime}(1) \mathbf{C}_{1}, \quad \mathbf{w}_{1}^{\prime}(2)=\sigma_{2} r_{1}(2) \mathbf{t}_{2}+r_{1}^{\prime}(2) \mathbf{C}_{2} .
\end{aligned}
$$




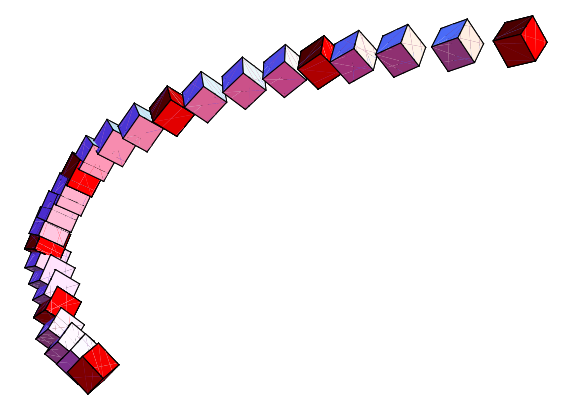

Fig. 2 Motion of a cube with seven interpolated positions.

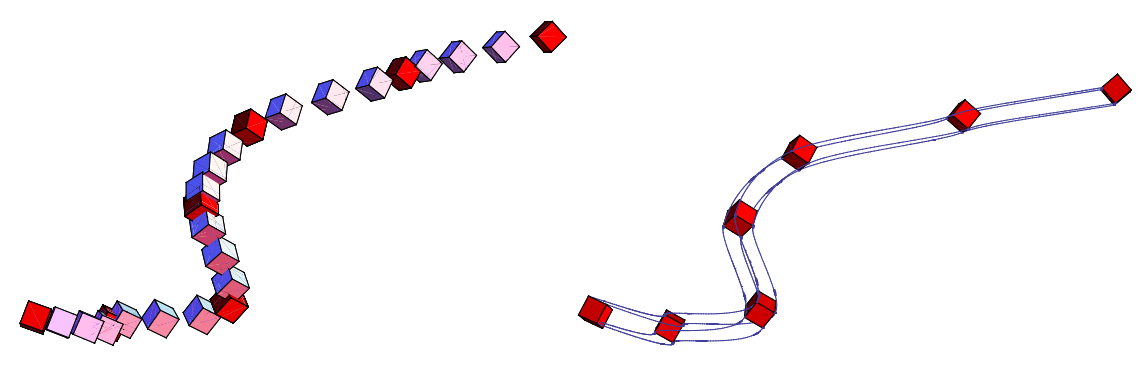

Fig. 3 Motion of a cube with seven interpolated positions (left) and the trajectories of corners of one side of the cube (right).

The presented scheme for $m=1$ is clearly entirely local. Consequently, for $m>1$, it can be applied on consecutive pairs of segments. This gives a $G^{1}$ rational quartic spline motion.

Let us demonstrate the performance of the presented interpolation scheme by some numerical examples.

Figure 2 shows the motion of a cube with seven interpolated positions. The input data were the positions of the center and the unit quaternions which corresponds to the rotations. The directions $\mathbf{t}_{j}$ were estimated using local parabolas (Bessel scheme), and the quaternion directions $\mathbf{u}_{j}$ were obtained as proposed in [9]. Figure 3 show another motion of a cube together with the trajectory of corners of one side of the cube.

\section{Conclusion}

This paper was devoted to piecewise rational motions with first order geometric continuity. As an application of these motions we described an interpolation scheme 
which generates rational spline motions of degree four from a sequence of given positions, some of which are complemented by associated tangent information. As demonstrated by the results, the use of geometric interpolation makes it possible to deal successfully with the uneven distribution of the degrees of freedom between the trajectory of the origin and the rotation part of the rational motions.

Future work will focus on the asymptotic behaviour of the interpolation scheme, where we plan to analyze the relation between the sampling density (i.e., the distance between the positions which are to be interpolated) and the behaviour and existence of the interpolating motion, and on the extension of these concepts to motions with higher order of smoothness.

\section{References}

1. Mørken, K., Scherer, K.: A general framework for high-accuracy parametric interpolation. Math. Comp. 66, no. 217, 237-260 (1997)

2. de Boor, C., Höllig, K., Sabin, M.: High accuracy geometric Hermite interpolation. Comput. Aided Geom. Design, 4, no. 4, 269-278 (1987)

3. Hoschek, J., Lasser D.: Fundamentals of Computer Aided Geometric Design. AK Peters, Wellesley Mass (1993)

4. Schröcker, H.-P., Jüttler, B.: Motion interpolation with Bennett biarcs. In Kecskemethy, A., Müller, A. (eds.) Proc. Computational Kinematics A. Springer, pp. 101-108 (2009)

5. Bottema, O., Roth, B.: Theoretical Kinematics. Dover (1990)

6. Jüttler, B., Wagner, M.G.: Kinematics and animation. In: G. Farin, J. Hoschek, and M.-S. Kim, editors, Handbook of Computer Aided Geometric Design, pp. 723-748. Elsevier, Amsterdam (2002)

7. Röschel, O.: Rational motion design-a survey. Comput. Aided Design 30, no. 3, 169-178 (1998)

8. Rath, W.: A kinematic mapping for projective and affine motions and some applications. In: Dillen, F. (ed.) et al. (Eds.) Geometry and topology of submanifolds, VIII. World Scientific. 292-301 (1996)

9. Horsch, T., Jüttler, B.: Cartesian spline interpolation for industrial robots. Comput. Aided Design, 30, 217-224 (1998)

10. Mørken, K.: Parametric interpolation by quadratic polynomials in the plane. In: Mathematical methods for curves and surfaces, pp. 385-402. Vanderbilt Univ. Press, Nashville (1995)

11. Kozak, J., Žagar, E.: On geometric interpolation by polynomial curves. SIAM J. Numer. Anal., 42, no. 3, 953-967 (2004) 